\title{
Factors affecting survival in 37 consecutive patients undergoing de novo stereotactic radiosurgery for contiguous sites of vertebral body metastasis from renal cell carcinoma
}

\author{
Jonathan N. Sellin, MD, William Reichardt, BA, Andrew J. Bishop, MD, Dima Suki, PhD, \\ Laurence D. Rhines, MD, Stephen H. Settle, MD, PhD, Paul D. Brown, MD, Jing Li, MD, PhD, \\ Ganesh Rao, MD, Eric L. Chang, MD, and Claudio E. Tatsui, MD \\ Departments of Neurosurgery and Radiation Oncology, The University of Texas MD Anderson Cancer Center, Houston, Texas
}

OBJECT Palliative resection of renal cell carcinoma (RCC) spinal metastasis is indicated in cases of neurological compromise or mechanical instability, whereas conventional external beam radiotherapy (EBRT) is commonly used for pain control. Recently, spinal stereotactic radiosurgery (SRS) has emerged as a safe alternative, delivering higher therapeutic doses of radiation to spinal metastases. To better understand factors affecting survival in patients undergoing spinal SRS for metastatic RCC, the authors performed a retrospective analysis of a consecutive series of cases at a tertiary cancer center.

METHODS Patients harboring contiguous sites of vertebral body involvement from metastatic RCC who received upfront spinal SRS treatment at The University of Texas MD Anderson Cancer Center between 2005 and 2012 were identified. Demographic data, pain scores, radiographic data, overall survival, complications, status of systemic disease, neurological and functional status, and time between primary diagnosis and diagnosis of metastasis (systemic and spinal) were analyzed to determine their influence on survival.

RESULTS Thirty-seven patients receiving treatment for 40 distinct, contiguous sites of disease were included. The median overall survival after spinal SRS was 16.3 months (range 7.4-25.3 months). Univariate analysis revealed several factors significantly associated with improved overall survival. Local progression after spinal SRS was associated with worse overall survival compared with sustained local control (HR 3.4, 95\% Cl 1.6-7.4, $p=0.002$ ). Median survival in patients with a Karnofsky Performance Scale (KPS) score $\geq 70$ was longer than in patients with a KPS score $<70$ (HR 4.7, $95 \% \mathrm{Cl} 2.1-10.7, p<0.001)$. Patients with neurological deficits at the time of spinal SRS had a shorter median survival than those without (HR 4.2, 95\% Cl 1.4-12.0, p = 0.008). Individuals with nonprogressive systemic disease at the time of spinal SRS had a longer median survival than those with systemic progression at the time of treatment $(\mathrm{HR} 8.3,95 \% \mathrm{Cl}$ 3.3-20.7, $p<0.001$ ). Median survival in patients experiencing any metastasis $<12$ months after primary RCC diagnosis was shorter than in patients experiencing any metastasis $>12$ months after primary diagnosis, a difference that approached but did not attain significance ( $\mathrm{HR} 1.9,95 \% \mathrm{Cl} 0.90-4.1, p=0.09)$. On multivariate analysis, local progression of disease after spinal SRS, metastasis $<12$ months after primary, KPS score $\leq 70$, and progression of systemic disease at time of spinal SRS all remained significant factors influencing survival (respectively, HR 3.7, $p=0.002 ; H R 2.6, p=$ 0.026 ; HR 4.0, $p=0.002$; and HR 13.2, $p<0.001$ ).

CONCLUSIONS We identified several factors associated with survival after spinal SRS for RCC metastases, including local progression, time between first metastasis and primary RCC diagnosis, KPS score, presence of neurological deficits, and progressive metastatic disease. These factors should be taken into consideration when considering a patient for spinal SRS for RCC metastases.

http://thejns.org/doi/abs/10.3171/2014.9.SPINE1482

KEY WORDS renal cell carcinoma; spinal metastasis; stereotactic radiosurgery; oncology

ABBREVIATIONS CTV = clinical target volume; EBRT = external beam radiotherapy; ESCC = epidural spinal cord compression; GTV = gross tumor volume; IMRT = intensity-modulated radiation therapy; KPS = Karnofsky Performance Scale; RCC = renal cell carcinoma; SINS = spinal instability neoplastic score; SRS = stereotactic radiosurgery; VAS = visual analog scale.

SUBMITTED January 22, 2014. ACCEPTED September 22, 2014.

INCLUDE WHEN CITING Published online October 31, 2014; DOI: 10.3171/2014.9.SPINE1482.

DISCLOSURE Dr. Rhines reports receiving teaching honoraria from Stryker and Globus. 
I $\mathrm{T}$ is estimated that up to $40 \%$ of patients with cancer will develop spinal metastasis at some point during their disease course. ${ }^{13}$ Management of spinal metastases usually requires a variety of treatment modalities, aiming at local disease control and symptom palliation. Common clinical presentations include pain from spinal instability or compression of neural structures, motor and sensory dysfunction, loss of sphincter control, and impaired ambulation. Traditionally, external beam radiotherapy (EBRT) has been used as a primary treatment modality for pain relief and local disease control. ${ }^{7,16}$ Medications, both analgesics for pain relief and chemotherapy for local control, have also been employed. Surgical intervention is palliative in nature and is usually reserved for instances of neurological compromise or gross spinal instability.

The efficacy of EBRT is limited by the radiosensitive nature of critical normal structures in close proximity to the spine, such as the spinal cord and small bowel, as this technique is not precise enough to deliver large doses of radiation to spinal lesions without endangering nearby neural or visceral structures. As a result, radiation doses delivered to lesions using EBRT are often insufficient to produce effective and durable tumor control. Stereotactic radiosurgery (SRS), on the other hand, because of its ability to produce a sharp dose fall-off at tumor margins, allows delivery of a high radiation dose to the tumor while sparing nearby critical normal structures, particularly the spinal cord. Although initially used to target intracranial lesions, technological advances have made SRS of the spine feasible. ${ }^{12}$ Gerszten et al. have demonstrated that spinal SRS is not only feasible, but also effective in improving clinical outcomes. ${ }^{11}$

The possibility of improved targeting and therapeutic tumor dosing provided by spinal SRS holds particular promise in the treatment of RCC metastases to the spine. Bone is the second most common site of metastasis for RCC, and the spine is the most frequent site of such bony metastases..$^{9,15}$ Pertinently, RCC is historically considered a radioresistant tumor, particularly in the setting of EBRT. 5,17,20,22,23 Even in the setting of spinal SRS, Gerszten et al. noted lower overall radiographic tumor control of RCC lesions in comparison with other histologies. ${ }^{11}$ Nevertheless, several series have demonstrated the clinical efficacy of spinal SRS in treating radioresistant RCC lesions and elucidated

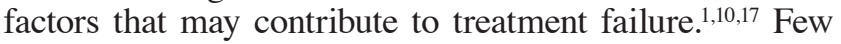
series, however, have focused on the impact of spinal SRS treatment of RCC metastases on overall survival.

\section{Methods \\ Patient Population}

The study population for this retrospective study comprised consecutive patients receiving initial spinal SRS treatment (i.e., first-time spinal radiosurgery, allowing for previous EBRT or medical interventions) for contiguous sites of spinal involvement of RCC metastases at The University of Texas MD Anderson Cancer Center from 2005 to 2013. All patients had a pathologically confirmed diagnosis of RCC and an MRI study documenting spinal metastases. Patients with high-grade epidural spinal cord compression (ESCC) or spinal instability, as judged by the multidisciplinary treatment team, were excluded. Patients with neurological deficits attributable to radiculopathy were, however, included. We defined a contiguous site of spinal involvement as a metastasis involving one or more contiguous vertebral levels affected by metastatic disease involving no more than 3 consecutive vertebral bodies. Hospital records and radiographic studies were reviewed. Data collected included patient demographics, date of primary RCC diagnosis, history of prior nephrectomy, Fuhrman grade of the nephrectomy specimen, date of diagnosis of first metastasis and its location, number of vertebral levels involved, date of diagnosis of spinal metastasis and its location, presence of neurological deficit prior to treatment, functional ability (Karnofsky Performance Scale [KPS] score), previous treatments (including chemo-, immuno-, and/or radiotherapy), pre-intervention pain (data available for all patients), post-intervention pain (data available for some patients only), pre-intervention scores on Bilsky's epidural spinal cord compression (ESCC) scale and the spinal instability neoplastic score (SINS), spinal SRS total dose (Gy) and fractions, status of extraspinal metastases at time of treatment, post-intervention radiographic occurrence of deformity or compression fracture, and need for further surgery at the spinal SRS index level. , $^{3,8}$

All patients in our series underwent restaging following the protocol of the genitourinary medical oncology service, with CT of the chest, abdomen, and pelvis obtained every 3-4 months. MRI of the brain or soft tissues was performed at the same intervals when metastatic disease affecting these locations was documented. We classified the status of extraspinal disease as none, concurrent, stable, or progressing. Concurrent disease was defined as metastases of any kind discovered at the same time as, or within 30 days of, the spinal metastasis. Stable disease was defined as preexisting metastases that had not changed in size or number in 2 consecutive pre-procedure imaging studies. Progressive disease was defined as preexisting metastases that had increased in size or number demonstrated by the latest preoperative imaging studies.

This study was approved by the MD Anderson Cancer Center clinical research committee for scientific integrity and the institutional review board.

\section{Prior EBRT or Surgery}

No patient in this series had received prior conventional radiotherapy to the lesion in question. Two patients had received conventional radiotherapy to lesions outside the spinal SRS field. No patient in this series had previously undergone surgery for the lesion in question. Two patients in this series had undergone previous vertebral augmentation procedures (kypho- or vertebroplasty) at index levels. These patients were included with the rationale that $\mathrm{ky}-$ pho- or vertebroplasty relieves symptoms but confers no oncological benefit.

\section{Spinal SRS Delivery Technique}

Our spinal SRS techniques have been described previously. ${ }^{17}$ Briefly, all patients underwent CT-based simulation. They were immobilized in an Elekta BodyFix stereotactic body frame system plastic fixation sheet. Diagnostic 
MRI scans were fused with simulation CT to best delineate the radiation targets and normal critical structures. The gross tumor volume (GTV) was defined as gross disease delineated by MRI scans and simulation CT scan. Clinical target volume (CTV) to encompass the microscopic disease is defined as the entire involved vertebral bodies up to and including the superior and inferior endplates, but excluding the disc and posterior elements unless they were involved with tumor. The treatment planning for spinal SRS was performed using Pinnacle (Philips Medical Systems). Intensity-modulated radiation therapy (IMRT) with coplanar beams was used. Treatment was delivered using a 21 EX linear accelerator equipped with a Millennium 120 multileaf collimator. Imaging guidance was used for each treatment session, which included an EXACT targeting system (Varian Medical Systems) and high-speed CT on rails (GE Medical Systems), all located in the same treatment suite. Orthogonal radiographs were obtained to further confirm the isocenter and accurate patient setup. The maximum allowable dose to any portion of the spinal cord was $10 \mathrm{~Gy}$. The radiation dose was typically prescribed to the $80 \%$ to $90 \%$ isodose line for CTV and to the $90 \%$ to $95 \%$ isodose line for GTV.

Per standard procedure, follow-up visits were scheduled at 3, 6, 9, and 12 months after treatment, then every 3-6 months thereafter. To analyze local progression (failure), we compared tumor progression, regression, or stability at the index level in all post-procedure MRIs with studies obtained immediately prior to spinal SRS.

\section{Dosing Regimen}

In this series, no patient had received any prior conventional radiation to the area to be targeted by spinal SRS. Four patients were treated with a total dose of 30 Gy delivered in 5 fractions, 9 individuals received 27 Gy delivered in 3 fractions, and the remaining 24 patients were treated to $24 \mathrm{~Gy}$ delivered in a single fraction.

The majority of patients, all of whom were radiation naïve at the treatment site, received 24 Gy in a single fraction. In patients whose treatment plan called for large radiation volumes or in cases in which lesions were close to critical normal structures, hypofractionated regimens were used as above, at the discretion of the radiation oncology team. In particular, in the early portion of our review period and the infancy of spinal SRS at our institution, the desire to be conservative and safe led to radiation delivery schemes using 5 doses.

\section{Data Analysis}

Frequencies and descriptive analyses of demographic and clinical variables were performed. The Kaplan-Meier method was used to estimate post-treatment survival, and survival curves were compared using a log-rank test. The Cox proportional hazards method was used to determine factors associated with survival after spinal SRS treatment at the univariate and multivariate levels. Hazard ratios and corresponding $95 \%$ confidence intervals were computed. A probability value $\leq 0.05$ was considered to be statistically significant. The data were analyzed using IBM SPSS version 21.

\section{Results}

Demographic and disease characteristics of the $37 \mathrm{pa}-$ tients who received initial spinal SRS treatment for contiguous sites of spinal involvement due to metastatic RCC are depicted in Table 1 . Three of the patients treated had more than 1 contiguous site of disease that received upfront spinal SRS, making for a total of 40 lesions treated.

\section{Overall Survival and Follow-Up}

The median overall survival after spinal SRS was 16.3 months (range 7.4-25.3 months). The median duration of follow-up was 49.0 months (range 38.2-75.8 months) among the 8 patients still alive at the time of the most recent scheduled follow-up. The following factors were not found to be significantly associated with survival: age, prior nephrectomy, Fuhrman grade, location of first metastasis, location of spinal metastasis treated, latency of spinal metastasis since RCC diagnosis or diagnosis of first metastasis, total number of vertebral levels involved, presence of solitary metastasis at time of spinal SRS, extraspinal disease burden, chemotherapy, and spinal SRS total dose and fractions.

TABLE 1. Patient demographic and disease characteristics, survival, and local control

\begin{tabular}{|c|c|}
\hline Parameter & Value \\
\hline \multicolumn{2}{|l|}{ Sex } \\
\hline Male & 28 \\
\hline Female & 9 \\
\hline \multicolumn{2}{|l|}{ Age at identification of primary tumor (yrs) } \\
\hline Median & 56.7 \\
\hline Range & $38-72.3$ \\
\hline \multicolumn{2}{|l|}{ Age at identification of metastasis (yrs) } \\
\hline Median & 62.2 \\
\hline Range & $42.4-72.8$ \\
\hline \multicolumn{2}{|l|}{ Location of spinal tumor } \\
\hline Cervical & 2 \\
\hline Thoracic & 13 \\
\hline Lumbosacral & 22 \\
\hline \multicolumn{2}{|l|}{ No. of VBs Involved } \\
\hline 1 & 22 \\
\hline 2 & 9 \\
\hline$>3$ & 6 \\
\hline \multicolumn{2}{|l|}{ Overall survival (mos) } \\
\hline Median & 16.3 \\
\hline Range & $7.4-25.3$ \\
\hline \multicolumn{2}{|l|}{ Follow-up (mos) } \\
\hline Median & 49.0 \\
\hline Range & $38.2-75.8$ \\
\hline \multicolumn{2}{|l|}{ Local progression } \\
\hline None & 21 \\
\hline Isolated & 4 \\
\hline W/ progression of systemic disease & 12 \\
\hline
\end{tabular}

$\mathrm{VB}=$ vertebral body. 


\section{Pre-Intevention and Post-Intervention Pain}

Pre-intervention pain data, as assessed on the 10-point visual analog scale (VAS), were available for all patients. The median preoperative pain score was 4 (range $0-10$ ). Post-intervention pain data were available for 29 of 37 patients. When available, the VAS score was collected variably at 1 month, 3 months, or 6 months post-proceduresome patients had data collected at multiple time points. Using the worst post-intervention VAS pain score of those available, as a conservative estimate, 12 patients reported improved pain levels post-intervention, 8 patients reported no change in their pain levels, and 9 patient reported worse pain (mean change in VAS score 0.28 , range -5 to 4 ).

\section{Levels Involved}

In our series, all patients received treatment for a contiguous site of disease. In $22(59.5 \%)$ of 37 cases the disease site involved a single vertebral body, in $9(24.3 \%)$ it involved 2 vertebral bodies, and in $6(16.2 \%)$ it involved 3 or more vertebral bodies.

\section{Radiographic Data}

Pre-intervention ESCC grades were collected for all 37 patients (harboring 40 lesions). Twenty-two of 40 lesions had Grade 1 epidural disease involvement, 18 lesions had Grade 0 epidural disease, and no patient had Grade 2 or 3 disease. Given that our cohort includes only patients selected for spinal SRS, it is not surprising that no cases of Grade 2 or 3 ESCC were encountered, as such patients would more likely present with a neurological deficit prompting surgery (Table 2 ).

Pre-intervention SINS were calculated for all 40 lesions treated. The mean SINS was 7.25 (range 3-11). Thirteen lesions were classified as stable (SINS 0-6) and 27 as indeterminate (SINS 7-12). No lesions were classified as unstable. Given that our cohort includes only patients selected for spinal SRS, it is understandable that none of the lesions analyzed qualified as frankly unstable per SINS criteria, as such lesions are more appropriate for surgical intervention (Table 2).

Follow-up spinal imaging was reviewed in all cases for the occurrence of compression fractures or kyphosis at the level of or attributable to the index lesions. Post-intervention, 11 lesions eventually developed compression fractures at an index level, 1 lesion developed a progressive kyphosis, and 2 lesions progressed to a combined compression fracture and kyphosis. Of note, 2 patients had received vertebroplasty or kyphoplasty for compression fracture at the index level prior to treatment-none of these patients went on to develop pathologic fracture or deformity (Table 2).

\section{Complications and Further Surgery}

In general, patients tolerated spinal SRS very well. One patient experienced some hair loss on the skin overlying the site of treatment. Two patients experienced nausea and vomiting requiring pharmacological therapy.

Two patients suffering from progressive disease went on to require kypho- or vertebroplasty at the index levelthese patients all suffered from painful compression fractures. Three patients suffering from progressive disease
TABLE 2. Pre-intervention and post-intervention radiographic parameters

\begin{tabular}{|c|c|}
\hline Parameter & No. of Lesions \\
\hline \multicolumn{2}{|l|}{ ESCC grade } \\
\hline 0 & 18 \\
\hline 1 & 22 \\
\hline 2 or 3 & 0 \\
\hline \multicolumn{2}{|l|}{ SINS, total } \\
\hline Stable (0-6) & 13 \\
\hline Indeterminate (7-12) & 27 \\
\hline Unstable (13-18) & 0 \\
\hline \multicolumn{2}{|l|}{ SINS, location } \\
\hline Junctional & 17 \\
\hline Mobile & 15 \\
\hline Semi-rigid & 8 \\
\hline Rigid & 0 \\
\hline \multicolumn{2}{|l|}{ SINS, pain } \\
\hline Mechanical & 5 \\
\hline Nonmechanical & 23 \\
\hline Pain free & 12 \\
\hline \multicolumn{2}{|l|}{ SINS, bone lesion quality } \\
\hline Blastic & 3 \\
\hline Mixed & 0 \\
\hline Lytic & 37 \\
\hline \multicolumn{2}{|l|}{ SINS, alignment } \\
\hline Normal alignment & 40 \\
\hline Subluxation/translation or de novo deformity & 0 \\
\hline \multicolumn{2}{|l|}{ SINS, VB collapse } \\
\hline$>50 \%$ & 0 \\
\hline$<50 \%$ & 4 \\
\hline No VB collapse but $>50 \%$ involvement & 18 \\
\hline None of above & 18 \\
\hline \multicolumn{2}{|l|}{ SINS, posterolateral involvement } \\
\hline Bilateral & 4 \\
\hline Unilateral & 25 \\
\hline None & 11 \\
\hline \multicolumn{2}{|l|}{ Post-intervention deformity } \\
\hline None & 26 \\
\hline Compression fracture & 11 \\
\hline Kyphosis & 1 \\
\hline Compression fracture + kyphosis & 2 \\
\hline
\end{tabular}

went on to require surgery, which involved oncological resection of tumor with anterior column reconstruction and posterolateral instrumentation and fusion. Two patients underwent kypho- or vertebroplasty followed by surgery. Of the 5 patients who required surgery or kypho/vertebroplasty and surgery, 1 patient had progression of dorsal epidural disease only, causing intractable pain and radiculopathy. The other 4 patients developed compression fractures secondary to progression of disease (captured on radiographic review as well), with intractable pain, radiculopathy, or myelopathy. 


\section{Local Control}

Local control was achieved in 21 patients in this series (57\%). Sixteen patients displayed radiographic disease progression. Local progression after spinal SRS was associated with worse overall survival compared with sustained local control (HR 3.44, 95\% CI 1.59-7.43, $\mathrm{p}=0.002$ ).

\section{Time Between First Metastasis and RCC Diagnosis}

The time between the initial diagnosis of metastasis of any kind and the diagnosis of primary disease was associated with the survival rate after spinal SRS. In 15 patients, metastasis was identified less than 12 months after the primary diagnosis of RCC; in the other 22 patients, metastases were identified more than 12 months after the primary diagnosis. The median overall survival after spinal SRS for the group with less than 12 months' latency was 14.9 months (95\% CI 10.6-19.3); in contrast, in the group of 14 patients who experienced metastases with more than 12 months' latency, the median overall survival was 44.1 months (95\% CI 17.5-70.7) after spinal SRS. On univariate analysis, the difference in median overall survival between the 2 groups approached but did not attain significance (HR 1.91, 95\% CI 0.90-4.1, p = 0.09).

\section{Karnofsky Performance Scale}

The Karnofsky Performance Scale (KPS) allows classification of patients according to the extent of their functional impairment. A KPS score $\geq 70$ implies that the patient is able to perform normal activities with few or no signs or symptoms of disease.

A KPS score $<70$ implies that the patient is in need of assistance to perform activities of daily living. A total of 26 patients had a KPS score $\geq 70$ prior to spinal SRS, and the remaining 11 patients had a KPS score $<70$. The median overall survival after spinal SRS for the patients with a KPS score $\geq 70$ was 23.4 months (95\% CI 14.4-32.4). The median overall survival for the patients with a KPS < 70 was 6.0 months (95\% CI 0.9-11.0). On univariate analysis, this difference in overall median survival was found to be statistically significant (HR 4.7, 95\% CI 2.1-10.7, p $<0.001)$.

\section{Neurological Deficit}

Of the 37 patients, 32 had no neurological deficits prior to spinal SRS. The remaining 5 patients had neurological deficits of varying severity (Frankel Grade B or C). The group without neurological deficits had a median overall survival of 22.1 months (95\% CI 12.3-31.9) after spinal SRS, whereas the group with neurological deficits had a median overall survival of 7.4 months (95\% CI 1.2-13.7) after spinal SRS. On univariate analysis, this difference in median overall survival between the 2 groups was statistically significant (HR 4.2, 95\% CI 1.4-12.0, $\mathrm{p}=0.008$ ).

\section{Status of Disease at the Time of Treatment}

We performed statistical analysis on lung, visceral, soft tissue, and extraspinal bony metastases and found that the presence of progressive disease significantly decreased overall survival for patients with each type of metastasis. Thus, for simplicity, analysis of extraspinal metastatic disease was condensed into two categories-progressive systemic disease or no progressive systemic disease. The presence of progressive systemic disease at the time of spinal SRS appeared to affect overall survival in patients with RCC. A total of 20 patients were found to have progressive disease at the time of spinal SRS treatment. The median overall survival of this group of patients was 7.4 months (95\% CI 1.7-13.2) after spinal SRS. The remaining 17 patients without progressive disease had a median overall survival of 77.4 months (95\% CI not defined) after spinal SRS. On univariate analysis, this difference was statistically significant (HR 8.31, 95\% CI 3.3-20.7, p < 0.001).

\section{Multivariate Analysis}

On multivariate analysis, several factors remained significantly associated with overall survival. Local disease progression after spinal SRS was associated with worse overall survival compared with local disease control (HR $3.7,95 \%$ CI $1.6-8.3, \mathrm{p}=0.002$ ). Diagnosis of metastasis less than 12 months after primary diagnosis was associated with worse overall survival compared to delayed time to metastasis (> 12 months), a difference which became significant on multivariate analysis (HR 2.6, 95\% CI 1.1$5.9, \mathrm{p}=0.026$ ). A KPS score $<70$ at the time of spinal SRS was associated with worse survival than a KPS score $\geq 70$ (HR 4.0, 95\% CI 1.7-10.0, $\mathrm{p}=0.002$ ). Finally, progression of systemic disease at time of spinal SRS was associated with worse overall survival than stable or concurrent disease (HR 13.2, 95\% CI 4.3-40.2, p < 0.001). The presence of a neurological deficit was not significantly associated with survival on multivariate analysis (Table 3).

Of note, none of the above factors found to significantly influence overall survival had a statistically significant influence on local control on univariate or subsequent multivariate analysis. Multivariate analysis, specifically, showed no significant increase in likelihood of local progression when comparing progression of systemic disease at time of spinal SRS to no progression (HR 2.0, 95\% CI 0.60-6.8, $\mathrm{p}=0.26$ ). Similarly, on multivariate analysis, latency $<12$ months from primary diagnosis to first metastasis compared with $>12$ months was not significantly associated with an increased risk of local progression (HR 1.7, 95\% CI $0.47-5.8, \mathrm{p}=0.43$ ), nor was KPS score at time of spinal SRS ( $<70$ vs $\geq 70)($ HR $1.5,95 \%$ CI $0.29-7.6, p=0.63)$, or the presence of neurological deficits at time of spinal SRS (neurological deficit compared with no neurological deficit) (HR 1.2, 95\% CI 0.15-8.9, p = 0.89) $($ Table 4).

TABLE 3. Factors influencing overall survival-multivariate analysis (hazard $=$ death)

\begin{tabular}{lccc}
\hline \multicolumn{1}{c}{ Parameter } & HR & $95 \% \mathrm{Cl}$ & p Value \\
\hline Local progression & 3.69 & $1.64-8.29$ & $0.002^{*}$ \\
\hline Latency $<12$ mos & 2.57 & $1.12-5.90$ & $0.026^{*}$ \\
\hline KPS score $<70$ & 4.0 & $1.67-10.0$ & $0.002^{*}$ \\
\hline Progression of systemic disease & 13.15 & $4.3-40.19$ & $<0.001^{*}$ \\
\hline Neurological deficit & 2.33 & $0.57-9.57$ & 0.24 \\
\hline
\end{tabular}

* Statistically significant. 
TABLE 4. Factors influencing local control-multivariate analysis (hazard $=$ recurrence)

\begin{tabular}{lccc}
\hline \multicolumn{1}{c}{ Parameter } & $\mathrm{HR}$ & $95 \% \mathrm{Cl}$ & $\mathrm{p} \mathrm{Value}$ \\
\hline Latency $<12$ mos & 1.66 & $0.47-5.80$ & 0.43 \\
\hline KPS score $<70$ & 1.50 & $0.29-7.61$ & 0.63 \\
\hline Progression of systemic disease & 2.02 & $0.60-6.82$ & 0.26 \\
\hline Neurological deficit & 1.16 & $0.15-8.86$ & 0.89 \\
\hline
\end{tabular}

\section{Discussion}

Over the past 2 decades, technological advances in the field of radiation oncology have made spinal SRS an appealing treatment option for patients with vertebral body metastases. Spinal SRS allows for precise targeting of therapeutic doses of radiation to spinal tumors, with a sharp fall-off in dosing outside the lesion that limits the exposure of neural structures to toxic doses of radiation, a concern that historically limited tumor dosing in spinal radiotherapy. Higher dosing is directly proportional to more effective treatment, allowing for better local control, pain relief, and improvement in quality of life., ${ }^{2,14}$

In a series of 500 patients, Gerszten et al. demonstrated that spinal SRS was safe and effective as a palliative intervention, without evidence of radiation-induced spinal cord injury and with improvement in pain control and radiographic tumor control. ${ }^{11}$ Subsequently, post-radiosurgical functional outcomes, complications, and overall survival times have been examined in large patient cohorts with heterogeneous primary histologies. ${ }^{6,18}$

Further study has focused specifically on the role of spinal SRS in the treatment of RCC metastasis to the spine, the promise of more therapeutic radiation dosing holding particular promise in the treatment of what was traditionally considered a radioresistant histology. ${ }^{5,17,20,22,23} \mathrm{RCC}$ metastases often progress despite EBRT, sometimes necessitating surgical intervention. Surgical management of RCC metastases, however, is associated with higher complication rates than the management of lesions of other, less hemorrhagic histological types. ${ }^{4}$

Literature review reveals 4 series to date that report on spinal SRS treatment of RCC spinal metastases (Table 5). Prior to publishing their broad case series, ${ }^{11}$ Gerszten and colleagues used descriptive statistics to evaluate clinical outcomes of SRS in a series of 48 patients with 60 RCC metastases to the spine..$^{10}$ Improvement in pain levels was noted in $89 \%$ of the 38 patients who were treated primarily for pain. Eight patients were treated primarily for tumor progression, and tumor control was achieved in $7(88 \%)$. However, 6 of 48 patients overall did eventually require surgical intervention for neurological compromise. Nguyen et al., similarly, assessed outcomes in a series of 48 patients with 55 lesions, showing an actuarial 1-year spine tumor progression-free survival rate of $82.1 \% .{ }^{17} \mathrm{Bal}-$ agamwala et al. reviewed their series of 57 patients (with 88 treatment sites) to identify factors associated with treatment failure. ${ }^{1}$ They reported median time to radiographic failure and pain progression of 26.5 and 26.0 months, respectively. Their multivariate analyses demonstrated that multilevel disease and neural foramen involvement correlated with radiographic failure. Staehler et al. reviewed a series of 106 patients with spinal or cerebral RCC metastases (55 spinal with 105 lesions, 51 cerebral) treated with SRS in combination with sorafenib or sunitinib (antiangiogenic agents). ${ }^{19}$ Their group reported a median survival of 17.4 months (range not available). On multivariate analysis, KPS score $>70$ was the only significant predictor of overall survival $(\mathrm{p}=0.006)$.

To our knowledge, however, our series is the first to detail factors associated with overall survival time in patients treated with spinal SRS exclusively for RCC spinal metastases. The paper by Staehler et al. does report prognostic factors influencing overall survival, but their cohort grouped cranial and spinal disease together. Furthermore, our series is the only one to analyze factors influencing overall survival in patients treated with upfront spinal SRS for RCC spinal metastases-none of our patients received previous conventional radiotherapy in the treated field-the Gerszten, Nguyen, and Balagamwala series all included patients who received prior radiotherapy (the Staehler paper does not comment on this factor). On univariate analysis, we found that in our group of patients treated with spinal SRS exclusively for spinal RCC metastatic disease, overall survival was significantly decreased in patients with local disease progression, a latency of spinal metastasis diagnosis $<12$ months or a KPS $<70$, neurological deficit, or progressive systemic disease at time of spinal metastasis diagnosis. On further multivariate analysis, local progression, latency $<12$ months, KPS $<$ 70 , and progressive systemic disease remained significant, with progressive systemic disease appearing to be most associated with poor survival (HR 13.15).

Intuitively, one might expect that healthy patients with indolent disease would live longer than sick patients with progressive disease. Our results are consistent with this notion-KPS score, the presence of neurological deficits, latency of diagnosis, and status of systemic disease are all

TABLE 5. Survival and local control/failure from RCC spinal SRS series to date

\begin{tabular}{lcccc}
\hline \multicolumn{1}{c}{ Authors \& Year } & $\begin{array}{c}\text { No. of Pts/ } \\
\text { No. of Treatments }\end{array}$ & $\begin{array}{c}\text { Median Survival in } \\
\text { Mos (range) }\end{array}$ & $\begin{array}{c}\text { Median Follow-Up in } \\
\text { Mos (range) }\end{array}$ & $\begin{array}{c}\text { No. of Treatment } \\
\text { Failures (\%) }\end{array}$ \\
\hline Gerszten et al., 2005 & $48 / 60$ & NA & $37(14-48)$ & $7(11.7)$ \\
\hline Nguyen et al., 2010 & $48 / 55$ & $22($ NA) & $13.1(3.3-54.5)$ & $12(21.8)$ \\
\hline Staehler et al., 2011 & $55 / 105$ & $17.4(\mathrm{NA})$ & $33.4(1-31)$ & NA \\
\hline Balagamwala et al., 2012 & $57 / 88$ & $8.3(1.5-26)$ & $5.4(0.3-38)$ & $20(22.7)$ \\
\hline Current series & $37 / 40$ & $16.3(7.4-25.3)$ & $49.0(38.2-75.8)$ & $16(43.3)$ \\
\hline
\end{tabular}

NA = data not available. 
likely significant because they serve as surrogates for the biology of a particular patient's cancer and general health status. Knowledge of these factors and their association with overall survival, however, remains important in influencing clinical decision making for patients with RCC. Interestingly, none of the factors associated with overall survival were significantly associated with local control. If certain clinical characteristics associated with worse overall survival do indeed represent surrogates for aggressive tumor biology-latency and progressive systemic disease, in particular-one might expect that the aggressive biology they signify would be conserved in the microenvironment of the spine and lead to more aggressive disease and local failures.

A recent retrospective review of patients undergoing resection for RCC spinal metastasis at the same institution (MD Anderson Cancer Center) also examined factors influencing overall survival. ${ }^{21}$ Survival analysis demonstrated that Fuhrman Grade 4 RCC, the presence of a preoperative neurological deficit, and progressive systemic disease were associated with worse overall survival, while exclusive spinal metastasis was associated with better overall survival. The median overall survival was 11.3 months, shorter than the median value observed in our series (16.3 months).

Certainly, any comparison of these 2 series has limitations; although collected at the same institution, data were not obtained in a head-to-head, prospective fashion. Furthermore, indications for surgical intervention-progressive neurological decline, spinal instability-differ from those for spinal SRS and generally result in more significant health limitations that may directly influence survival, as is likely represented by the significance of preoperative neurological deficits. It is worth noting, however, that the presence of progressive systemic disease as a harbinger of poor survival is conserved in both series and reinforces the notion that aggressive tumor biology leads to worse outcomes, regardless of intervention type, which might guide choice of therapy. Cautious comparison between the 2 series may lend some insight to and further refine patient selection based on expected overall survival, directing some patients toward aggressive surgical intervention while sparing patients with more limited expected survival the morbidity associated with aggressive resection.

\section{Conclusions}

Although the overall prognosis associated with metastatic RCC to the spine remains poor, tumor biology and physiology can differ from patient to patient. These differences yield variability in malignant behavior, prognosis, and thus treatment options appropriate for individual patients. We retrospectively analyzed data from a cohort of patients receiving spinal SRS treatment for RCC metastasis and identified several factors that may aid in predicting which patients will live longer and benefit most from first-time spinal SRS treatment. Of note, these same factors were not significantly associated with local failure. To better individualize treatment of metastatic RCC, physicians should take these factors into account prior to considering treatment regimens to ensure that patients receive maximal benefit from spinal SRS, a palliative procedure.

\section{References}

1. Balagamwala EH, Angelov L, Koyfman SA, Suh JH, Reddy CA, Djemil T, et al: Single-fraction stereotactic body radiotherapy for spinal metastases from renal cell carcinoma. Clinical article. J Neurosurg Spine 17:556-564, 2012

2. Bartelink H, Horiot JC, Poortmans PM, Struikmans H, Van den Bogaert W, Fourquet A, et al: Impact of a higher radiation dose on local control and survival in breast-conserving therapy of early breast cancer: 10 -year results of the randomized boost versus no boost EORTC 22881-10882 trial. J Clin Oncol 25:3259-3265, 2007

3. Bilsky M, Smith M: Surgical approach to epidural spinal cord compression. Hematol Oncol Clin North Am 20:13071317,2006

4. Bowers TA, Murray JA, Charnsangavej C, Soo CS, Chuang VP, Wallace S: Bone metastases from renal carcinoma. The preoperative use of transcatheter arterial occlusion. J Bone Joint Surg Am 64:749-754, 1982

5. Cannady SB, Cavanaugh KA, Lee SY, Bukowski RM, Olencki TE, Stevens GH, et al: Results of whole brain radiotherapy and recursive partitioning analysis in patients with brain metastases from renal cell carcinoma: a retrospective study. Int J Radiat Oncol Biol Phys 58:253-258, 2004

6. Chao ST, Koyfman SA, Woody N, Angelov L, Soeder SL, Reddy CA, et al: Recursive partitioning analysis index is predictive for overall survival in patients undergoing spine stereotactic body radiation therapy for spinal metastases. Int J Radiat Oncol Biol Phys 82:1738-1743, 2012

7. Faul CM, Flickinger JC: The use of radiation in the management of spinal metastases. J Neurooncol 23:149-161, 1995

8. Fisher CG, DiPaola CP, Ryken TC, Bilsky MH, Shaffrey CI, Berven SH, et al: A novel classification system for spinal instability in neoplastic disease: an evidence-based approach and expert consensus from the Spine Oncology Study Group. Spine (Phila Pa 1976) 35:E1221-E1229, 2010

9. Frank I, Blute ML, Cheville JC, Lohse CM, Weaver AL, Zincke H: An outcome prediction model for patients with clear cell renal cell carcinoma treated with radical nephrectomy based on tumor stage, size, grade and necrosis: the SSIGN score. J Urol 168:2395-2400, 2002

10. Gerszten PC, Burton SA, Ozhasoglu C, Vogel WJ, Welch WC, Baar J, et al: Stereotactic radiosurgery for spinal metastases from renal cell carcinoma. J Neurosurg Spine 3:288295, 2005

11. Gerszten PC, Burton SA, Ozhasoglu C, Welch WC: Radiosurgery for spinal metastases: clinical experience in 500 cases from a single institution. Spine (Phila Pa 1976) 32:193-199, 2007

12. Hamilton AJ, Lulu BA, Fosmire H, Stea B, Cassady JR: Preliminary clinical experience with linear accelerator-based spinal stereotactic radiosurgery. Neurosurgery 36:311-319, 1995

13. Klimo P Jr, Schmidt MH: Surgical management of spinal metastases. Oncologist 9:188-196, 2004

14. Kong FM, Ten Haken RK, Schipper MJ, Sullivan MA, Chen M, Lopez C, et al: High-dose radiation improved local tumor control and overall survival in patients with inoperable/ unresectable non-small-cell lung cancer: long-term results of a radiation dose escalation study. Int J Radiat Oncol Biol Phys 63:324-333, 2005

15. Levy DA, Slaton JW, Swanson DA, Dinney CP: Stage specific guidelines for surveillance after radical nephrectomy for local renal cell carcinoma. J Urol 159:1163-1167, 1998

16. Markoe AM, Schwade JG: The role of radiation therapy in the management of spine and spinal cord tumors, in Rea GL (ed): Spine Tumors. Park Ridge, IL: American Association of Neurological Surgeons, 1994, pp 23-35

17. Nguyen QN, Shiu AS, Rhines LD, Wang H, Allen PK, Wang $\mathrm{XS}$, et al: Management of spinal metastases from renal cell 
carcinoma using stereotactic body radiotherapy. Int J Radiat Oncol Biol Phys 76:1185-1192, 2010

18. Sheehan JP, Shaffrey CI, Schlesinger D, Williams BJ, Arlet $\mathrm{V}$, Larner J: Radiosurgery in the treatment of spinal metastases: tumor control, survival, and quality of life after helical tomotherapy. Neurosurgery 65:1052-1062, 2009

19. Staehler M, Haseke N, Nuhn P, Tüllmann C, Karl A, Siebels $\mathrm{M}$, et al: Simultaneous anti-angiogenic therapy and singlefraction radiosurgery in clinically relevant metastases from renal cell carcinoma. BJU Int 108:673-678, 2011

20. Sundaresan N, Scher H, DiGiacinto GV, Yagoda A, Whitmore W, Choi IS: Surgical treatment of spinal cord compression in kidney cancer. J Clin Oncol 4:1851-1856, 1986

21. Tatsui CE, Suki D, Rao G, Kim SS, Salaskar A, Hatiboglu MA, et al: Factors affecting survival in 267 consecutive patients undergoing surgery for spinal metastasis from renal cell carcinoma. Clinical article. J Neurosurg Spine 20:108116,2014

22. Weber K, Doucet M, Kominsky S: Renal cell carcinoma bone metastasis-elucidating the molecular targets. Cancer Metastasis Rev 26:691-704, 2007
23. Weber KL, Doucet M, Price JE: Renal cell carcinoma bone metastasis: epidermal growth factor receptor targeting. Clin Orthop Relat Res (415 Suppl):S86-S94, 2003

\section{Author Contributions}

Conception and design: Tatsui, Sellin, Rhines. Acquisition of data: Tatsui, Reichardt, Bishop, Rhines, Settle, Brown, Li, Rao, Chang. Analysis and interpretation of data: Tatsui, Sellin. Drafting the article: Sellin. Critically revising the article: Tatsui, Sellin, Rao. Reviewed submitted version of manuscript: Tatsui. Statistical analysis: Suki. Study supervision: Tatsui, Sellin, Rhines.

\section{Correspondence}

Claudio E. Tatsui, 1515 Holcombe Blvd., Unit 442, Houston, TX 77303. email: cetatsui@mdanderson.org. 